\title{
Special issue on formal methods for security engineering
}

\section{Antonella Santone ${ }^{1}$}

Published online: 11 September 2018

C Springer-Verlag France SAS, part of Springer Nature 2018

This special issue presents papers from the First Workshop on FORmal methods for Security Engineering (ForSE), which was held on February 2017 in Porto, in conjunction with ICISSP, the $3^{\text {th }}$ International Conference on Information Systems Security and Privacy. Due to the constant increase in computing capabilities, every aspect of society has been redefined by software systems. However, the lack of proper security testing has made them vulnerable to a rising number of cyber attacks. Formal methods represent an excellent tool to model such systems mathematically and verify their security properties to improve their resilience against malicious code. Still, there is a large space for collaboration between the formal methods and security communities to address such essential security problems. The ForSE workshop aims at closing this gap and foster the development of verifiable secure and malware resistant systems.

The ForSE workshop has received 16 paper submissions coming from 9 different countries around the world (Austria, Bahrain, France, Germany, Italy, Lebanon, Netherlands, South Africa, USA).
Based on ForSE 2017 review reports and discussions during and following the workshop, authors of seven papers were invited to submit revised and expanded versions of their workshop paper for this special issue. After the JICV's traditional peer-review process, three papers were accepted.

As editor of this special issue I am very grateful for the efforts made by the authors to push the quality of their contributions to a high level during the review process. Also, I would like to express my gratitude to the reviewers for their constructive feedbacks, the Editor-in-Chief, Prof. Eric Filiol, for his guidance and support and the members of the editorial team for their help with producing this issue.
Antonella Santone

antonella.santone@unimol.it

1 Department of Bioscience and Territory, University of Molise, Pesche, IS, Italy 